\title{
ON THE NORMAL SPECTRUM OF A SUBNORMAL OPERATOR
}

\author{
J. W. BUNCE AND J. A. DEDDENS ${ }^{1}$
}

\begin{abstract}
In this note we present a new characterization for subnormality which is purely $C^{*}$-algebraic. We also establish an intrinsic characterization of the normal spectrum for a subnormal operator, which enables us to prove that $\sigma_{\perp}(\pi(S)) \subseteq \sigma_{\perp}(S)$ for any *-representation $\pi$.
\end{abstract}

An operator $A$ on a Hilbert space $\mathcal{H}$ is called subnormal if there exists a normal operator $N$ on a larger Hilbert space $\mathscr{K} \supseteq \mathcal{K}$ such that $A=N \mid \mathcal{H}$. P. R. Halmos [4] introduced subnormal operators and showed that there is always an essentially unique minimal normal extension.

Following M. B. Abrahamse and R. G. Douglas [1] we define the normal spectrum $\sigma_{\perp}(S)$ of a subnormal operator $S$ to be the spectrum of the minimal normal extension of $S$. J. Bram [2] continued the study of subnormal operators and showed that subnormality is preserved under *-representations and that $\partial \sigma(S) \subseteq \sigma_{\perp}(S) \subseteq \sigma(S)$. In this note we use Bram's results to give a new characterization for subnormality that is purely $C^{*}$-algebraic. It easily follows that subnormality is preserved by ${ }^{*}$-representations. We also give an intrinsic characterization of the normal spectrum $\sigma_{\perp}(S)$, from which it easily follows that $\sigma_{\perp}(\pi(S)) \subseteq \sigma_{\perp}(S)$ for any ${ }^{*}$-representation $\pi$ of $C^{*}(S)$. This answers problem 2 in [1] in the affirmative. For any operator $T, C^{*}(T)$ will denote the $C^{*}$-algebra generated by $T$.

Proposition 1. An operator $A$ on a Hilbert space $\mathcal{H}$ is subnormal if and only if $\sum_{i, j=0}^{n} B_{i}^{*} A^{* j} A^{i} B_{j} \geqslant 0$ for every finite set

$$
B_{0}, B_{1}, B_{2}, \ldots, B_{n} \in C^{*}(A)
$$

Proof. It follows from [4] and Theorem 1 in [2] that $A$ is subnormal if and only if $\sum_{i, j=0}^{n}\left(A^{i} f_{j}, A^{j} f_{i}\right) \geqslant 0$ for every finite set of vectors $f_{0}, f_{1}, \ldots, f_{n} \in \mathcal{H}$. For $B_{0}, B_{1}, \ldots, B_{n} \in C^{*}(A)$ and $x \in \mathcal{H}$, let $f_{i}=B_{i} x$. We see that if $A$ is subnormal, then

Received by the editors July 3, 1976.

AMS (MOS) subject classifications (1970). Primary 47B20, 47A10; Secondary 46L05.

Key words and phrases. Subnormal operator, normal spectrum, $C^{*}$-algebra.

${ }^{1}$ The work of both authors was partially supported by the National Science Foundation Grant \# MCS 73-08540. 


$$
\begin{aligned}
0 & \leqslant \sum_{i, j=0}^{n}\left(A^{i} f_{j}, A^{j} f_{i}\right)=\sum_{i, j=0}^{n}\left(A^{i} B_{j} x, A^{j} B_{i} x\right) \\
& =\sum_{i, j=0}^{n}\left(B_{i}^{*} A^{* j} A^{i} B_{j} x, x\right)=\left\{\left\{\sum_{i, j=0}^{n} B_{i}^{*} A^{* j} A^{i} B_{j}\right\} x, x\right]
\end{aligned}
$$

for every $x \in \mathcal{H}$. Hence $0 \leqslant \sum_{i, j=0}^{n} B_{i}^{*} A^{* j} A^{i} B_{j}$. Conversely, let $f_{0}, f_{1}, \ldots$, $f_{n} \in \mathcal{H}$ be given. It clearly suffices to assume that all the $f_{j}$ 's lie in a subspace Tl cyclic for $C^{*}(A)$, with cyclic vector $x_{0}$. Then there exist $B_{k j} \in C^{*}(A)$ with $f_{j}=\lim _{k \rightarrow \infty} B_{k j} x_{0}$. Hence

$$
\begin{aligned}
\sum_{i, j=0}^{n}\left(A^{i} f_{j}, A^{j} f_{i}\right) & =\lim _{k \rightarrow \infty}\left(\sum_{i, j=0}^{n}\left(A^{i} B_{k j} x_{0}, A^{j} B_{k i} x_{0}\right)\right) \\
& =\lim _{k \rightarrow \infty}\left(\sum_{i, j=0}^{n} B_{k i}^{*} A^{* j} A^{i} B_{k j} x_{0}, x_{0}\right) \geqslant 0
\end{aligned}
$$

and $A$ is subnormal.

CorollaRy 1 (BRAM). If $S$ is subnormal and $\pi$ is $a^{*}$-representation of $C^{*}(S)$ then $\pi(S)$ is also subnormal.

We now give an intrinsic characterization of the spectrum $\sigma_{\perp}(S)$ of the minimal normal extension $N$ of a subnormal operator $S$. Write $\rho_{\perp}(S)=$ $\mathrm{C} \backslash \sigma_{\perp}(S)$.

PROPOSITION 2. If $S$ is subnormal then $\lambda \in \rho_{\perp}(S)$ if and only if there exists $\alpha>0$ such that

$$
\sum_{i, j=0}^{n} B_{i}^{*} S^{* j}(S-\lambda)^{*}(S-\lambda) S^{i} B_{j} \geqslant \alpha \sum_{i, j=0}^{n} B_{i}^{*} S^{* j} S^{i} B_{j}
$$

for every finite set $B_{0}, B_{1}, \ldots, B_{n} \in C^{*}(S)$.

Proof. Recall that for a normal operator the spectrum is equal to the approximate point spectrum. Thus $\lambda \in \rho_{\perp}(S) \Leftrightarrow \lambda \in \rho(N) \Leftrightarrow N-\lambda$ is bounded below $\Leftrightarrow$ there exists $\alpha>0$ with $\|(N-\lambda) y\|^{2} \geqslant \alpha\|y\|^{2}$ for all $y \in \mathscr{K}$. Since $N$ is the minimal normal extension of $S, \mathcal{K}$ is the smallest reducing subspace for $N$ containing $\mathscr{H}$. Thus $\mathscr{K}=\operatorname{span}\left\{N^{* i} x_{0}: x_{0} \in \mathcal{H}\right.$, $i \geqslant 0\}$; thus $\lambda \in \rho_{\perp}(S) \Leftrightarrow$ there exists $\alpha>0$ with $\left\|(N-\lambda) \sum_{i=0}^{n} N^{* i} f_{i}\right\|^{2} \geqslant$ $\alpha\left\|\sum_{i=0}^{n} N^{* i} f_{i}\right\|^{2}$, for every finite set $f_{0}, f_{1}, f_{2}, \ldots, f_{n} \in \mathcal{H}$. We may rewrite this last inequality as

$$
\left((N-\lambda) \sum_{j=0}^{n} N^{* j} f_{j},(N-\lambda) \sum_{i=0}^{n} N^{* i} f_{i}\right) \geqslant \alpha\left(\sum_{j=0}^{n} N^{* j} f_{j}, \sum_{i=0}^{n} N^{* i} f_{i}\right)
$$

or (since $N$ is normal, $N$ and $N^{*}$ commute)

$$
\sum_{i, j=0}^{n}\left((N-\lambda) N^{i} f_{j},(N-\lambda) N^{j} f_{i}\right) \geqslant \alpha \sum_{i, j=0}^{n}\left(N^{i} f_{j}, N^{j} f_{i}\right)
$$

or (since $S=N \mid \mathcal{H C})$ 


$$
\sum_{i, j=0}^{n}\left((S-\lambda) S^{i} f_{j},(S-\lambda) S^{j} f_{i}\right) \geqslant \alpha \sum_{i, j=0}^{n}\left(S^{i} f_{j}, S^{j} f_{i}\right) .
$$

Thus $\lambda \in \rho_{\perp}(S)$ if and only if (2) holds for every finite set $f_{0}, f_{1}, \ldots, f_{n} \in \mathcal{H}$. If we let $f_{i}=B_{i} x$ for $B_{i} \in C^{*}(S)$ and $x \in \mathcal{H}$ we obtain

$$
\left(\sum_{i, j=0}^{n}\left(B_{i}^{*} S^{* j}(S-\lambda)^{*}(S-\lambda) S^{i} B_{j}\right) x, x\right) \geqslant \alpha\left(\sum_{i, j=0}^{n} B_{i}^{*} S^{* j} S^{i} B_{j} x, x\right)
$$

which is just (1). To see that (1) implies (2), it again suffices to assume that all the $f_{i}$ lie in a subspace cyclic for $C^{*}(S)$. The proof then follows precisely as in the proof of Proposition 1.

COROLlaRY 2. If $S$ is subnormal and $\pi$ is a ${ }^{*}$-representation of $C^{*}(S)$ then $\sigma_{\perp}(\pi(S)) \subseteq \sigma_{\perp}(S)$.

Proof. It follows immediately from Proposition 2 that $\rho_{\perp}(S) \subseteq \rho_{\perp}(\pi(S))$. The conclusion now follows by taking complements.

Remark 1. It is easily seen that Propositions 1 and 2 remain valid when the condition that $B_{i} \in C^{*}(A)$ is replaced by either the condition $B_{i} \in \mathscr{B}(\mathcal{H})$ or by the condition $B_{i} \in \mathcal{P}\left(A, A^{*}\right)=$ the set of all noncommutative polynomials in $A$ and $A^{*}$. In fact, one need only require that $B_{i} \in \mathscr{B}(A)$ where $\mathscr{B}(A)$ is any subset such that any cyclic subspace for $C^{*}(A)$ is also cyclic for $\mathscr{B}(A)$. For example, if $A$ itself is cyclic one need only require $B_{i} \in \mathscr{P}(A)$, or if $A^{*}$ is cyclic one need only require that $B_{i} \in \mathscr{P}\left(A^{*}\right)$.

REMARK 2. It follows from Corollary 2 that if two subnormal operators are algebraically equivalent (i.e., there exists a faithful representation $\pi$ of $C^{*}\left(S_{1}\right)$ onto $C^{*}\left(S_{2}\right)$ with $\left.\pi\left(S_{1}\right)=S_{2}\right)$, then their minimal normal extensions are also algebraically equivalent.

REMARK 3. The normal spectrum $\sigma_{\perp}(S)$ behaves well under direct sums $\left(\sigma_{\perp}\left(S_{1} \oplus S_{2}\right)=\sigma_{\perp}\left(S_{1}\right) \cup \sigma_{\perp}\left(S_{2}\right)\right)$, but unlike the spectrum it is not upper semicontinuous. Consider the weighed shifts $S_{n}$ with weights $\sqrt{n /(n+1)}$, $\sqrt{(n+1) /(n+2)}, \sqrt{(n+2) /(n+3)}, \ldots$ respectively. Then (see [3]) each $S_{n}$ is subnormal with $\sigma_{\perp}\left(S_{n}\right)=$ closed unit disk, and $S_{n}$ converges uniformly to the unilateral shift $U_{+}$with $\sigma_{\perp}\left(U_{+}\right)=$unit circle.

Remark 4. Proposition 1 enables one to give $C^{*}$-algebraic proofs for certain properties of subnormal operators, the proofs of which normally involve the normal extension. For example, if $A$ is subnormal then $A$ is hyponormal. (Proof. Take $n=1$ and $B_{0}=-A^{*}, B_{1}=I$.) If $A$ is an invertible subnormal then $A^{-1}$ is subnormal. (Proof. For $D_{0}, D_{1}, \ldots, D_{n} \in$ $C^{*}\left(A^{-1}\right)=C^{*}(A)$ set $B_{i}=A^{-n} D_{n-i}$; then

$$
\begin{aligned}
0 & \leqslant \sum_{i, j=0}^{n} B_{i}^{*} A^{* j} A^{i} B_{j}=\sum_{i, j=0}^{n} D_{n-i}^{*} A^{*-n} A^{* j} A^{i} A^{-n} D_{n-j} \\
& =\sum_{i, j=0}^{n} D_{n-i}^{*}\left(A^{*-1}\right)^{n-j}\left(A^{-1}\right)^{n-i} D_{n-j}
\end{aligned}
$$


and the obvious change of variables yields the desired result.)

\section{REFERENCES}

1. M. B. Abrahamse and R. G. Douglas, A class of subnormal operators related to multiply-connected domains, Advances in Math. 19 (1976), 106-148.

2. J. Bram, Subnormal operators, Duke Math J. 22 (1955), 75-94. MR 16, 835.

3. R. Gellar and L. J. Wallen, Subnormal weighted shifts and the Halmos-Bram criterion, Proc. Japan Acad. 46 (1970), 375-378. MR 51 \#8879.

4. P. R. Halmos, Normal dilations and extensions of operators, Summa Brasil. Math. 2 (1950), 125-134. MR 13, 359.

Department of Mathematics, University of Kansas, LaWrence, Kansas 66045 